\title{
Model-Updated Image-Guided Minimally Invasive Off-Pump Transcatheter Aortic Valve Implantation
}

\author{
Mohamed Esmail Karar ${ }^{1}$, Matthias John², David Holzhey ${ }^{3}$, Volkmar Falk ${ }^{4}$, \\ Friedrich-Wilhelm Mohr ${ }^{1,3}$, and Oliver Burgert ${ }^{1}$ \\ ${ }^{1}$ Innovation Center Computer Assisted Surgery (ICCAS), University of Leipzig, Germany \\ ${ }^{2}$ Siemens AG, Healthcare Sector, Forchheim, Germany \\ ${ }^{3}$ Department of Cardiac Surgery, Heart Center, University of Leipzig, Germany \\ ${ }^{4}$ Division of Heart and Vascular Surgery, University Hospital Zurich, Switzerland \\ mohamed.karar@medizin.uni-leipzig.de
}

\begin{abstract}
This paper presents a method for assisting the placement of stented aortic valve prosthesis during minimally invasive off-pump transcatheter aortic valve implantation (TAVI) under live 2-D X-ray fluoroscopy guidance. The proposed method includes a dynamic overlay of an intra-operative 3-D aortic root mesh model and an estimated target area of valve implantation onto live 2D fluoroscopic images. This is based on a template-based tracking procedure of a pigtail catheter without further injections of contrast agent. Minimal userinteraction is required to initialize the algorithm and to correct fluoroscopy overlay errors if needed. Retrospective experiments were carried out on ten patient datasets from the clinical routine of the TAVI. The mean displacement errors of the updated aortic root mesh model overlays are less than $2.0 \mathrm{~mm}$ without manual overlay corrections. The results show that the guidance performance of live 2-D fluoroscopy is potentially improved when using our proposed method for the TAVIs.
\end{abstract}

Keywords: Transcatheter aortic valve implantation, X-ray fluoroscopy, imageguided interventions, aortic valve prosthesis.

\section{Introduction}

Aortic valve replacement is the standard treatment of degenerative aortic valve stenosis. However, many elderly patients have an elevated predicted operative risk that could compromise the patient's outcome after standard open heart surgery [1]. Transcatheter aortic valve implantation (TAVI) therefore presents a good alternative to the standard surgical treatment for elderly and high-risk patients with severe aortic stenosis [1].

The transapical TAVI is a recent and minimally invasive, off-pump technique that consists of a left anterolateral mini-thoracotomy for direct antegrade surgical access through the apex of the left ventricle [2]. This is followed by the insertion of an inflatable bioprosthetic valve through a catheter and the implantation within the diseased, native aortic valve under rapid ventricular pacing (RVP). During the intervention, the placement of stented aortic valve prosthesis such as the Edwards 
Sapien $^{\mathrm{TM}}$ THV stent-valve (Edwards Lifesciences, Irvine, USA) is crucial and is typically performed under two-dimensional (2-D) X-ray fluoroscopy guidance [1-3].

Live 2-D fluoroscopy displays 2-D projection images of interventional devices such as intra-cardiac catheters, aortic valve prosthesis (AVP) and some anatomical structures. Cardiac blood vessels, such as the aortic root and coronary arteries, are only visible in single-plane fluoroscopic images if the contrast agent is injected using a pigtail catheter for guiding the TAVI [2]. However, the dosages of contrast injections must be minimized because of renal insufficiencies in elderly patients. Before starting the TAVI procedure, the surgeon can use an angiographic C-arm system to reconstruct intra-operative three-dimensional (3-D) CT-like images of the aortic root under RVP from an acquired rotational 2-D fluoroscopic image sequence with 15-25 ml contrast agent of $200^{\circ}$ over 5 seconds [3], see Fig. 1. This small amount of contrast agent is equivalent to just a single angiogram aortic root shot.

There are few previous studies related to image-guided TAVI interventions. In [4], an intra-operative guidance system has been proposed to include the planning system of [5] and to perform real-time tracking of the AVP in fluoroscopic images. A system for automatic segmentation and for the static overlay of aortic root volume and landmarks on live fluoroscopic images is described in [6,7]. Using intra-operative MRI guidance, a new robotic assistance system has been evaluated for delivering the AVP using a phantom [8].

In this paper, we aim to automatically display an appropriate placement position of the transcatheter AVP using live 2-D fluoroscopy guidance. Based on the motion of a pigtail catheter in the absence of contrast agent, the proposed method approximates the motion of the aortic root to update the overlay of a 3-D aortic root mesh model including anatomical valve landmarks such as coronary ostia from intra-operative 3-D C-arm CT images onto 2-D fluoroscopic images. If the contrast agent appears in fluoroscopic images, this fluoroscopy overlay is not required and can be switched off [9]. Furthermore, automatic estimation of a target area of implantation is visualized to potentially improve the accuracy of the implanted AVP.
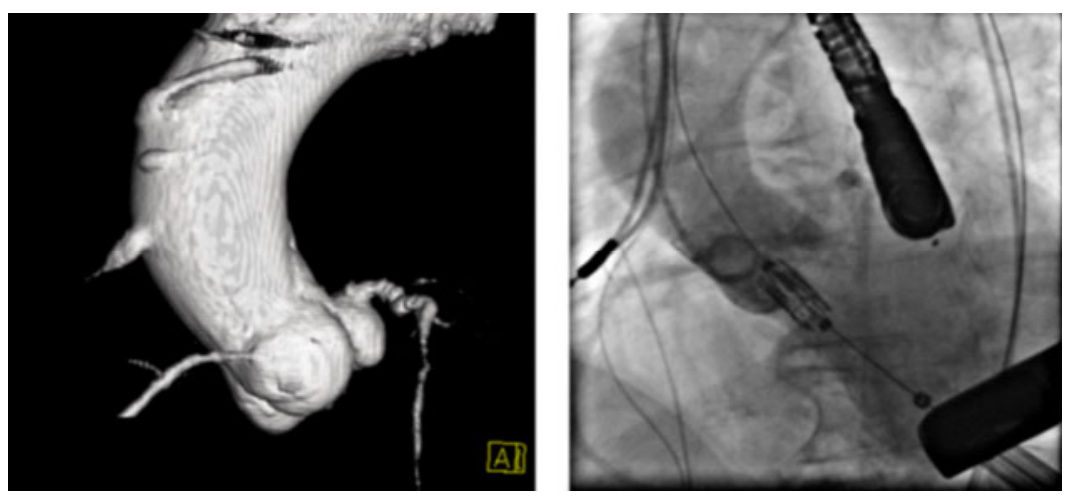

Fig. 1. Intra-operative 3-D C-arm CT image of the aortic root (left) and 2-D fluoroscopy guidance for assisting the placement of aortic valve prosthesis (right) 


\section{Method}

The proposed method starts with algorithm initialization, followed by an updating procedure of the overlaid aortic root model. In the initialization step, a 3-D geometrical mesh model of the aortic root with valve landmarks is acquired from the interventional $\mathrm{C}$-arm system. Based on the best experience and knowledge of the physician, a target area of valve implantation is automatically estimated inside the 3$\mathrm{D}$ mesh model. The aortic root model and the target area of valve implantation are then projected and manually aligned onto a contrast image. The contrast image is a fluoroscopic image which is automatically detected to display the aortic root roadmap with contrast agent. Finally, the overlay of the aortic root model and the target area of implantation onto live fluoroscopic images are updated by following the aortic root motion via the tracking of a pigtail catheter.

In the following, each component of this proposed method is described in more detail.

\subsection{Aortic Root Mesh Model and Target Area of Implantation}

In order to guide the TAVI procedure, a 3-D triangulated mesh model of the aortic root has been used. The mesh geometry is generated based on a learning-based 3-D boundary detector of the aortic root in intra-operative C-arm CT images [6]. Using a discriminative learning-based landmark detector, the resulting model moreover includes eight anatomical valve landmarks [6]; namely two points of coronary ostia (left $\mathrm{LCO}_{3 d}$ and right $R C \mathrm{O}_{3 d}$ ), three points of commissures (left $L C_{3 d}$, right $R C_{3 d}$ and non-coronary $N C_{3 d}$ ) and the three lowest points (hinge points) of each leaflet cusp (left $L L C_{3 d}$, right $R L C_{3 d}$ and non-coronary $N L C_{3 d}$ ), see Fig. 2 (left) .

Based on professional surgical experience, the correct placement of AVP should be one-third to one-half of its length above and perpendicular to the aortic annulus [2]. In this study, a target area of valve implantation is defined by two embedded circles of annulus and ostia planes with the normal center line to the valve annulus [5]. The annulus circle $A C_{3 d}=C\left(c_{a}, r_{a}\right)$ is defined by the three lowest points of the valve cusps. The circle's center $c_{a}$ is the centroid of the cusp points $\left(L L C_{3 d}, R L C_{3 d}, N L C_{3 d}\right)$. The radius $r_{a}$ is calculated from the lengths of the sides of the cusp points $(a, b, c)$ and the area of three cusps points $A_{a}$ from heron's formula:

$$
r_{a}=\frac{a b c}{4 A_{a}}, \text { where } A_{a}=\sqrt{\frac{\left(a^{2}+b^{2}+c^{2}\right)^{2}-2\left(a^{4}+b^{4}+c^{4}\right)}{4}} .
$$

The circle is inscribed to the corresponding plane of cusp points through $c_{a}$ normal to

$$
n_{a}=\frac{\left(c_{a}-L L C_{3 d}\right) \times\left(c_{a}-R L C_{3 d}\right)}{\left\|c_{a}-L L C_{3 d}\right\| \cdot\left\|c_{a}-R L C_{3 d}\right\|} .
$$

Similarly, the ostia circle $O C_{3 d}$ is calculated at the level of the lowest coronary ostium within the aortic root and parallel to the $A C_{3 d}$. The normal center line $C L_{3 d}$ is connected between the two centers of $A C_{3 d}$ and $O C_{3 d}$ as shown in Fig. 2 (left). 


\subsection{Alignment of Aortic Root Model onto Contrast Image}

By analyzing the histogram of live fluoroscopic images and using the 98-percentile as a threshold measurement of contrast agent, a contrast image is automatically detected after learning the histogram feature curve of the first 20 frames of the fluoroscopic sequence without contrast agent [9]. In this contrast image, the aortic root roadmap shows up in dark pixels as depicted in Fig. 2 (middle).

The 3-D aortic mesh model with landmarks and the target area of implantation are then projected onto a 2-D fluoroscopy plane by using the transformation matrix of the interventional $\mathrm{C}$-arm system. This projected data is initially aligned with the aortic root roadmap onto the contrast image by the physician, as shown in Fig. 2 (right).
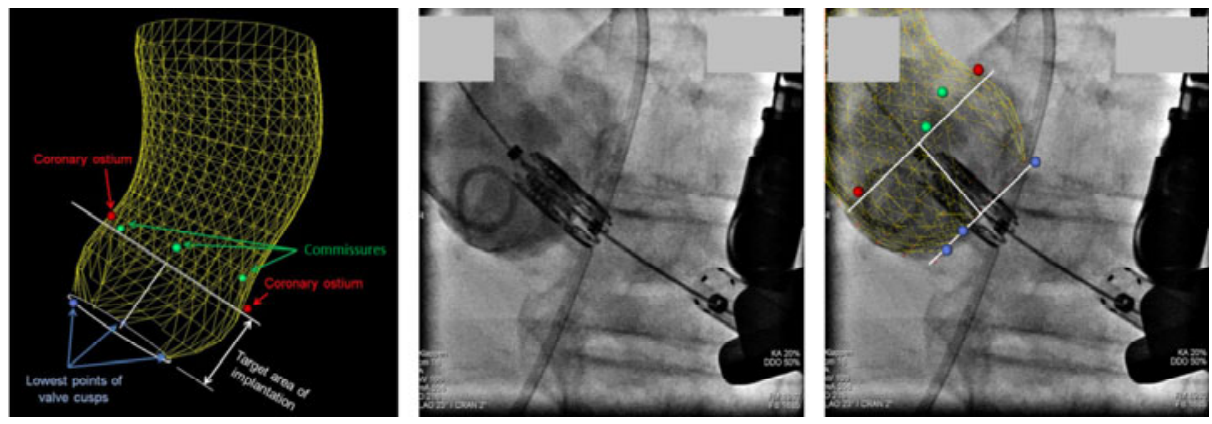

Fig. 2. 3-D aortic root mesh model including anatomical valve landmarks and target area of implantation (left), a contrast image shows up the aortic root roadmap with contrast agent (middle), and the manual alignment of projected aortic mesh model and target area of implantation with the aortic roadmap onto the contrast image (right)

\subsection{Updating Aortic Root Model Overlay}

The collaborating physicians confirmed that the aortic root moves with the pigtail catheter unless it is pulled or pushed manually. Therefore, we assumed the imagebased tracking procedure of the pigtail catheter to provide a dynamic overlay of the projected aortic mesh model with landmarks and the target area of implantation onto live 2-D fluoroscopy as follows. Firstly, fluoroscopic images are preprocessed using a 2-D Gabor filter [10], in order to reduce the image noise and to enhance the features of the pigtail catheter. Secondly, a template matching approach [11] is applied to determine the current position of the pigtail catheter within each fluoroscopic image of the live sequence, see Fig. 3 (left). The template image of the pigtail catheter $t$ is manually defined on the first image of the sequence. A region of interest (ROI) is defined to reduce the processing time and increase the algorithm robustness. In practice, the size of the ROI is chosen to be $2.5 x$ the size of the template image.

In this matching approach, $I(x, y)$ denotes the intensity of a preprocessed ROI image of the size $S_{x} \times S_{y}$ at point $(x, y), x \in\left\{0, \ldots, S_{x}-1\right\}, y \in\left\{0, \ldots, S_{y}-1\right\}$ and the template image $t$ of the size $s_{x} \times s_{y}$. The position of the catheter is determined by a pixelwise comparison of the ROI image with the template image, based on the computing of fast 
normalized cross correlation (FNCC) coefficient $\gamma$ at each point $(u, v)$ for ROI and for template images. Eq. 3 provides the definition of $\gamma$. Here $\bar{i}_{u, v}$ and $\bar{t}$ are the mean brightness values within the ROI and the template image respectively. The normalized value $\gamma_{\max }$ at the point $(u, v)$ in the current ROI image defines the best matching location of the template.

$$
\gamma(u, v)=\frac{\sum_{x, y}\left(I(x, y)-\bar{i}_{u, v}\right)(t(x-u, y-v)-\bar{t})}{\sqrt{\sum_{x, y}\left(I(x, y)-\bar{i}_{u, v}\right)^{2} \sum_{x, y}(t(x-u, y-v)-\bar{t})^{2}}} .
$$

Finally, the translational motion of the visualized aortic root model, valve landmarks and the target area of implantation is then updated by calculating the displacement $\mathbf{d}=$ $\left(d_{x}, d_{y}\right)$ of pigtail catheter between two images of the sequence [12]. This is based on the different matching positions of the catheter template between these two images. In order to ensure the high accuracy of the tracked pigtail catheter, the template-based tracking algorithm can be temporarily stopped if the best matching value of $\gamma_{\max }$ is less than $50 \%$ in the current proceeded image.
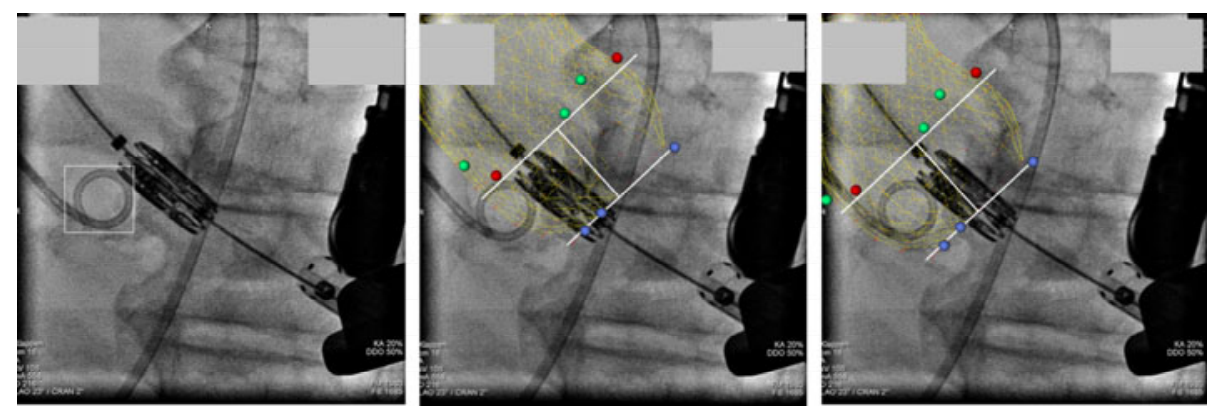

Fig. 3. Template matching of a pigtail catheter (left), projected aortic mesh model with landmarks and target area of implantation onto a fluoroscopic image of live sequence with static overlay (middle) and with updating model overlay (right)
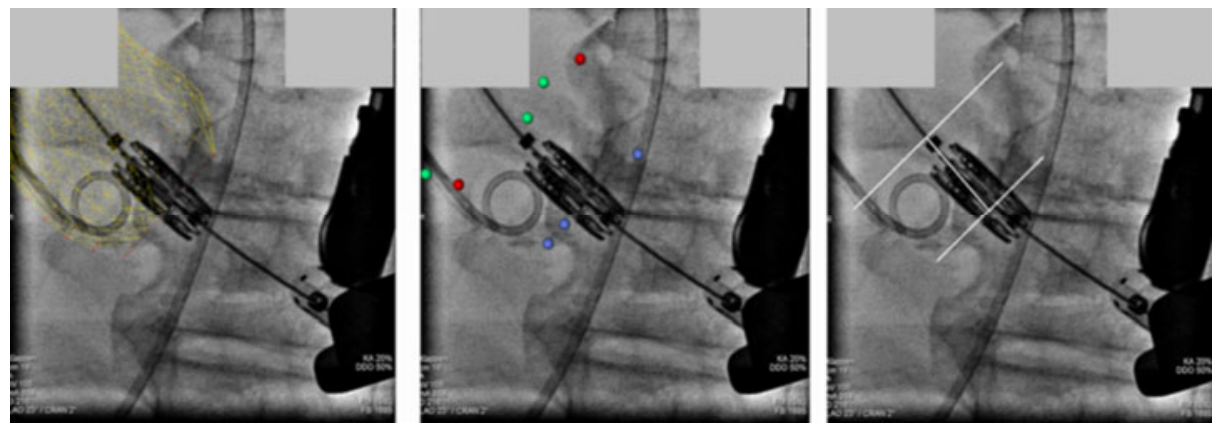

Fig. 4. Views of visualized aortic mesh model (left), valve landmarks (middle), and target area of implantation (right) projected onto a fluoroscopic image 


\subsection{User Interaction and Visualization}

An interactive graphical user interface (GUI) is integrated within the proposed method based on $\mathrm{C}++$ and open source components such as the Visualization Toolkit (VTK) [13]. The parameter values needed to compute the 3-D to 2-D transformation matrix of the C-arm imaging system are imported from a Digital Imaging and Communications in Medicine (DICOM) file or given by the user. Moreover, different views of the overlaid mesh model, valve landmarks and target area of implantation are separately visualized to allow the physician to display only the required information for the prosthesis deployment, as depicted in Fig. 4.

\section{Results and Evaluation}

In order to test and evaluate the proposed method, experiments were retrospectively carried out on different patient datasets from the clinical routine of the TAVI. These datasets included ten fluoroscopic image sequences and related aortic root mesh models of three female and two male patients around 84 years of age. Each fluoroscopic sequence included 90 images with $512 \times 512$ to $1024 \times 1024$ pixels. The pixel size was approximately $0.15 \mathrm{~mm}$. All fluoroscopic images and aortic root models were acquired from the interventional $\mathrm{C}$-arm system (Artis zeego, Siemens AG, Healthcare Sector, Forchheim, Germany) at the Heart Center Leipzig, Germany.

Using a standard PC with Intel CPU $(2.4 \mathrm{GHz})$, the total computation times of the algorithm initialization and dynamic overlay procedures were approximately three to five minutes and 100 to 125 milliseconds per frame respectively.

The updating performance of overlaid aortic root model onto live fluoroscopic images was indirectly evaluated by calculating the absolute mean and maximum displacement errors, $d_{\text {mean }} \pm$ standard deviation (SD) and $d_{\text {max }}$, between the expected and the computed displacements of the pigtail template over all tested fluoroscopic images, see Fig. 5. The expected displacements of the tracked catheter are manually performed by an experienced user during the evaluation procedure only. The evaluation results did not include the images with high doses of contrast agent, because these contrasted images (three to ten images per each sequence) can temporarily stop the template-based tracking algorithm of the pigtail catheter.

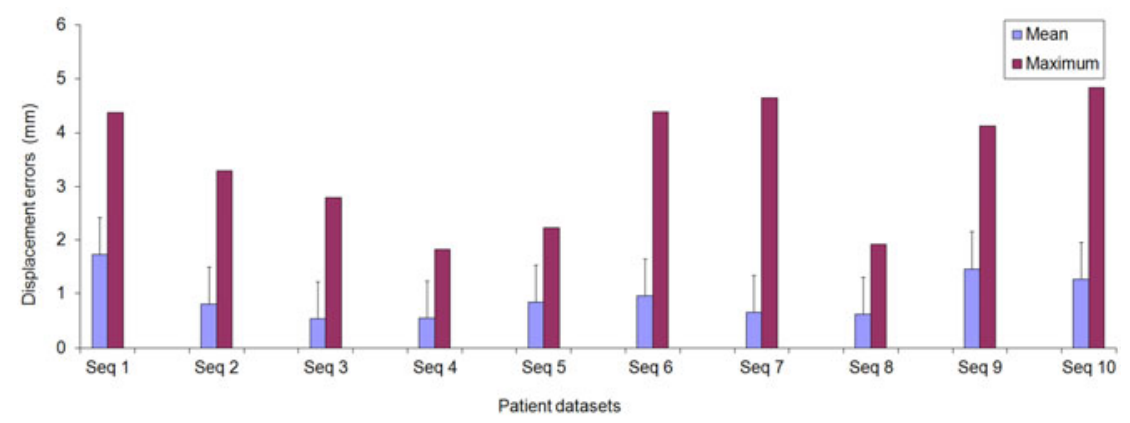

Fig. 5. Evaluation results of the updated aortic root model overlay onto fluoroscopic image sequences for ten patient datasets based on displacement errors of the pigtail catheter 
As depicted in Fig. 5, the fluoroscopic image sequence (seq. 1) shows relatively high displacement errors of $d_{\text {mean }}=1.73 \pm 0.86 \mathrm{~mm}$ and $d_{\text {max }}=4.37 \mathrm{~mm}$. This is due to the pigtail catheter having been slightly pulled by the physician.

Due to an overlapping of the pigtail catheter with the prosthesis, image sequences 7 and 10 yielded the highest maximum displacement errors $4.65 \mathrm{~mm}$ and $4.84 \mathrm{~mm}$, respectively, but have such large errors for only one to three images per sequence. However, all tested fluoroscopic images showed that the absolute mean displacement errors are less than $2.0 \mathrm{~mm}$ and within the clinically accepted ranges.

\section{Discussion}

We have proposed a method to assist the placement of the AVP during minimally invasive off-pump TAVI under 2-D fluoroscopy guidance. Compared to the work in [7], this proposed method can avoid misalignments between the static overlay of the aortic root model and invisible aortic root roadmaps in live fluoroscopic images during the TAVI procedure. Furthermore, automatic definition and visualization of the target area of implantation is potentially saving the physician's effort and surgery time as well.

In order to perform dynamic overlay of the aortic root model and the target area of implantation onto live fluoroscopic images, the template matching using FNCC approach has been applied for tracking the pigtail catheter which approximates the translational motion of the aortic root without contrast agent injections. Interventional devices such as the transesophageal echocardiography (TEE) probe and guide wires have no effect on the detection of the pigtail catheter, because they often lie outside of the ROI. Overlapping with the AVP may affect the matching accuracy of the pigtail catheter. Nevertheless, the obtained results demonstrated that our method is robust enough to track the pigtail catheter in the context of the TAVI procedure.

Unfortunately a clinical validation of this study would require additional contrast agent injections which are dangerous for elderly and high-risk patients who have renal insufficiencies. Because there are no tools or ground truth datasets to accurately identify the correct overly of the aortic root model without contrast injections, the updating accuracy of overlaid aortic root models has therefore been indirectly evaluated by estimating the displacement errors of the pigtail catheter for all ten patient datasets, see Fig. 5. The collaborating physicians assumed that the displacement errors for the TAVI were 2 to $5 \mathrm{~mm}$, and that the margin of errors should not exceed $2 \mathrm{~mm}$ in narrow calcific aortic stenosis. In Fig. 5, the evaluation results show that the absolute mean errors are approximately less than $2.0 \mathrm{~mm}$ within the clinically accepted ranges. An interactive GUI has been developed to manually correct the high displacement errors by adding suitable offset values in X-Y directions for the tracked pigtail catheter.

Using this image-based method to guide the TAVI procedure, an assistance system in a "hybrid" operating room is under development which is connected to the interventional $\mathrm{C}$-arm system and is capable of real-time clinical studies.

Acknowledgments. The Authors would like to thank Alois Nöttling and Stefan Ammon for the technical support during this study. This work is supported by German Academic Exchange Service (DAAD) under scholarship number A0690520. This work is partially funded by Siemens AG, Forchheim, Germany. 


\section{References}

1. Ferrari, E., Sulzer, C., Marcucci, C., Qanadly, S.D., Locca, D., Berdajs, D., Von Segesser, L.K.: Transcatheter Aortic Valve Implantation (TAVI): State of The Art Techniques and Future Perspectives. Swiss. Med. Wkly. 140, w13127 (2010)

2. Walther, T., Dewey, T., Borger, M.A., Kempfert, J., Linke, A., Becht, R., Falk, V., Schuler, G., Mohr, F.W., Mack, M.: Transapical Aortic Valve Implantation: step by step. Ann. Thorac. Surg. 87, 276-283 (2009)

3. Kempfert, J., Falk, V., Schuler, G., Linke, A., Merk, D., Mohr, F.W., Walther, T.: DynaCT During Minimally Invasive Off-Pump Transapical Aortic Valve Implantation. Ann. Thorac. Surg. 88, 2041 (2009)

4. Karar, M.E., Gessat, M., Walther, T., Falk, V., Burgert, O.: Towards a New Image Guidance System for Assisting Transapical Minimally Invasive Aortic Valve Implantation. In: Conf. Proc. IEEE Eng. Med. Biol. Soc., pp. 3645-3648. IEEE Press, New York (2009)

5. Gessat, M., Merk, D.R., Falk, V., Walther, T., Jacobs, S., Nottling, A., Burgert, O.: A Planning System for Transapical Aortic Valve Implantation. In: Proc. of SPIE Medical Imaging (2009)

6. Zheng, Y., John, M., Liao, R., Boese, J., Kirschstein, U., Georgescu, B., Zhou, S.K., Kempfert, J., Walther, T., Brockmann, G., Comaniciu, D.: Automatic Aorta Segmentation and Valve Landmark Detection in C-Arm CT: Application to Aortic Valve Implantation. In: Jiang, T., Navab, N., Pluim, J.P.W., Viergever, M.A. (eds.) MICCAI 2010. LNCS, vol. 6361, pp. 476-483. Springer, Heidelberg (2010)

7. John, M., Liao, R., Zheng, Y., Nottling, A., Boese, J., Kirschstein, U., Kempfert, J., Walther, T.: System to Guide Transcatheter Aortic Valve Implantations Based on Interventional C-arm CT Imaging. In: Jiang, T., Navab, N., Pluim, J.P.W., Viergever, M.A. (eds.) MICCAI 2010. LNCS, vol. 6361, pp. 375-382. Springer, Heidelberg (2010)

8. Ming, L., Kapoor, A., Mazilu, D., Horvath, K.A.: Pneumatic Actuated Robotic Assistant System for Aortic Valve Replacement Under MRI Guidance. IEEE Trans. on Biomed. Eng. 58, 443-451 (2011)

9. Condurache, A.P., Aach, T., Eck, K., Bredno, J.: Fast Detection and Processing of Arbitrary Contrast Agent Injections in Coronary Angiography and Fluoroscopy. In: Proc. of Bildverarbeitung fuer die Medizin (2004)

10. Kong, W.K., Zhang, D., Li, W.: Palmprint Feature Extraction Using 2-D Gabor Filters. J. Pattern Recognition 36, 2339-2347 (2003)

11. Briechle, K., Hanebeck, U.D.: Template Matching Using Fast Normalized Cross Correlation. In: Proc. of SPIE Optical Pattern Recognition XII (2001)

12. Atasoya, S., Groher, M., Darko, Z., Glocker, B., Waggershauser, T., Pfister, M., Navab, N.: Real-Time Respiratory Motion Tracking: Roadmap Correction for Hepatic Artery Catheterizations. In: Proc. of SPIE Medical Imaging (2008)

13. Visualization Toolkit, Kitware Inc., http : / / www. vtk. org 